\title{
Cryptic Translocation Identification in Human and Mouse Using Several Telomeric Multiplex FISH (TM-FISH) Strategies
}

\author{
Octavian Henegariu, Sevilhan Artan, John M. Greally, Xiao-Ning Chen, \\ Julie R. Korenberg, Gail H. Vance, Lisa Stubbs, Patricia Bray-Ward, and \\ David C. Ward
}

Department of Genetics (OH, SA, JMG, PB-W, DCW), Yale University School of Medicine, New Haven, Connecticut; Departments of Pediatrics and Human Genetics (X-NC, JRK), Cedars-Sinai Medical Center, Los Angeles, California; Department of Medical Genetics (GHV), Indiana University, Indianapolis, Indiana; and Lawrence Livermore National Laboratory (LS), Livermore, California

\begin{abstract}
SUMMARY: Experimental data published in recent years showed that up to $10 \%$ of all cases of mild to severe idiopathic mental retardation may result from small rearrangements of the subtelomeric regions of human chromosomes. To detect such cryptic translocations, we developed a "telomeric" multiplex fluorescence in situ hybridization (M-FISH) assay, using a set of previously published and commercially available subtelomeric probes. This set of probes includes $41 \mathrm{cosmid} / \mathrm{PAC} / \mathrm{P} 1 \mathrm{clones}$ located from less than 100 kilobases to approximately 1 megabase from the end of the chromosomes. Similarly, a published mouse probe set, comprised of BACs hybridizing to the closest known marker toward the centromere and telomere of each mouse chromosome, was used to develop a mouse-specific "telomeric" M-FISH. Three different combinatorial labeling strategies were used to simultaneously detect all human subtelomeric regions on one slide. The simplest approach uses only three fluors and can be performed in laboratories lacking sophisticated imaging equipment or personnel highly trained in cytogenetics. A standard fluorescence microscope equipped with only three filters is sufficient. Fluor-dUTPs and labeled probes can be custom made, thus dramatically reducing costs. Images can be prepared using imaging software (Adobe Photoshop) and analysis performed by simple visual inspection. (Lab Invest 2001, 81:483-491).
\end{abstract}

\begin{abstract}
I $\mathrm{t}$ is estimated that $7.4 \%$ of all cases of mild to severe idiopathic mental retardation result from small rearrangements of the subtelomeric regions of human chromosomes (Flint et al, 1995; Knight et al, 1999; Slavotinek et al, 1999). Rearrangements involving fewer than 1 to 2 megabases $(\mathrm{Mb})$ usually are undetectable by cytogenetic banding techniques or molecular painting methods such as multiplex fluorescence in situ hybridization (M-FISH) and spectral karyotyping (Azofeifa et al, 2000; Schrock et al, 1996; Speicher et al, 1996). Such small translocations or deletions are easier and more conveniently detected by FISH, using subtelomeric chromosome-specific probes (Bacino et al, 2000; Ballif et al, 2000; Ghaffari et al, 1998; Granzow et al, 2000; Knight et al, 1997). Using a set of previously published probes, we developed three different telomeric multiplex FISH (TMFISH) strategies that allow simultaneous hybridization of all 41 subtelomeric probes on one slide. These commercially available probes (American Type Culture
\end{abstract}

Received January 22, 2001.

Address reprint requests to: Dr. Octavian Henegariu, Microarray Facility, $M B \mho B$ Department, Yale University School of Medicine, WWW 5, 333 Cedar Street, New Haven, CT 06510. E-mail: octavian. henegariu@yale.edu
Collection [ATCC], Rockville, Maryland) include previously published cosmids, $\mathrm{P} 1$ and $\mathrm{P} 1$ artificial chromosome (PAC) clones (National Institutes of Health and Institute of Molecular Medicine, 1996). All these probes are known to be located at distances between 100 kilobases $(\mathrm{kb})$ and $1 \mathrm{Mb}$ from the end of the chromosomes. We also developed a similar M-FISH approach for mouse chromosomes, using a set of bacterial artificial chromosome (BAC) probes (Korenberg et al, 1999) located at the centromeric and distal telomeric ends of the telocentric mouse chromosome. These probes correspond to most telomeric and centromeric markers known on mouse chromosomes. We refer to this as mouse TM-FISH (mTM-FISH) to differentiate it from a true TM-FISH approach, as described for the human chromosomes. Although the physical distance between each probe in the set and its corresponding chromosome end is not yet known, this mouse set can be used for mapping purposes or in screening for chromosomal aberrations in murine embryonic stem cells (Henegariu et al, 2001a). Because TM-FISH uses commercially available probes (ATCC and Research Genetics, Huntsville, Alabama), they can be prepared and labeled in any laboratory at a cost per analysis significantly less than regular M-FISH. The various fluor-deoxyuridine triphosphates (dUTPs) used for probe labeling can also be custom 

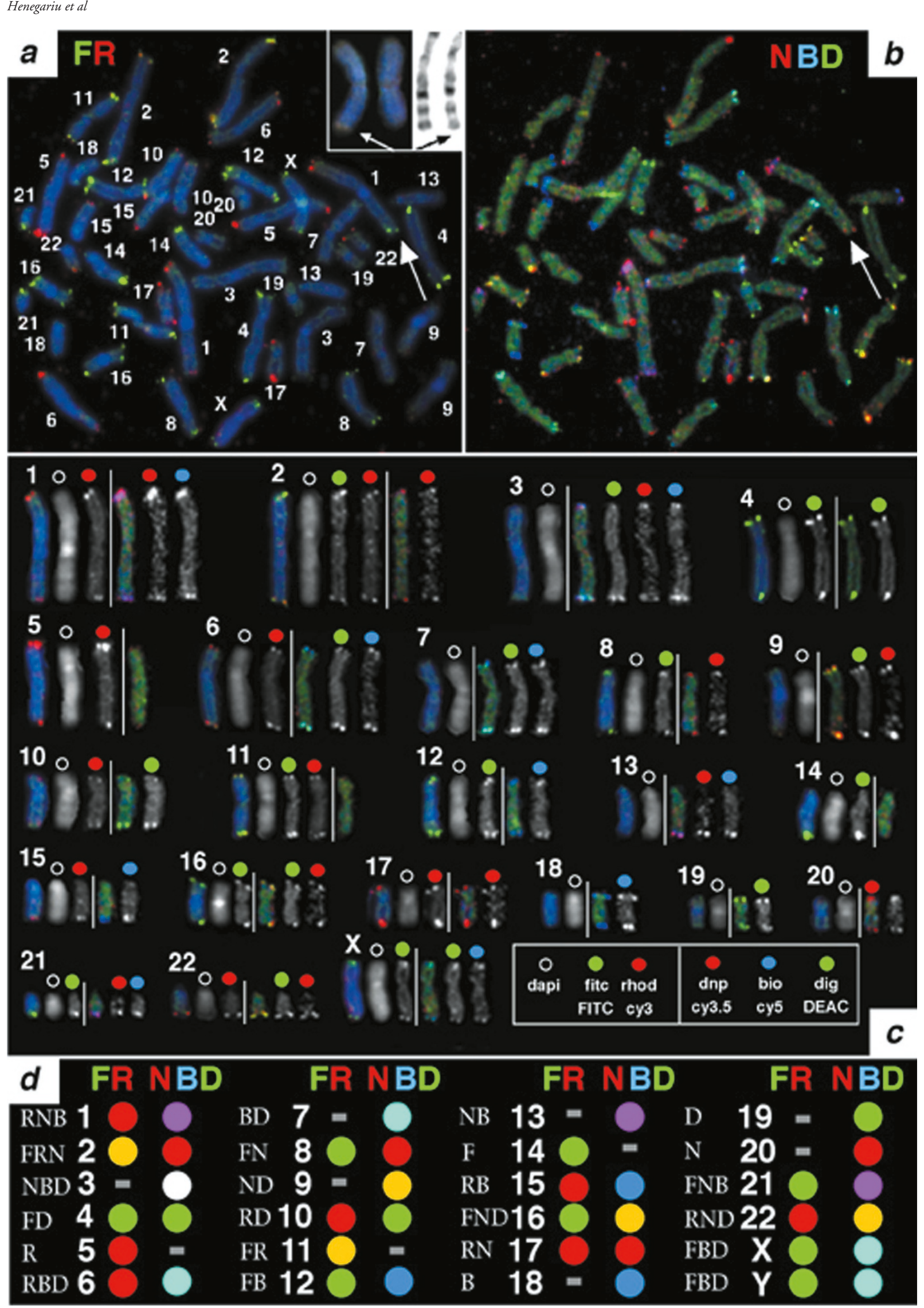

Figure 1. 
prepared in any laboratory at a very reduced cost (Henegariu et al, 2000). TM-FISH can be performed without the need for specialized hardware or software and with increased sensitivity of detection of small translocations compared with regular $\mathrm{M}-\mathrm{FISH}$.

\section{Results and Discussion}

Simultaneous use of 40 (mouse) or 41 (human) unique probes in $\mathrm{FISH}$ raised problems not seen with chromosome-painting probes. The human set included clones which yielded weaker fluorescent signals (especially for chromosomes $5,10 p, 11,16,19$, 20 , and $X Y$ ). Most of these were cosmids, which carry smaller inserts than a BAC or PAC. Two labeling procedures were tested and compared, nick translation and degenerate oligonucleotide priming PCR (DOP-PCR), each with distinct advantages and disadvantages. In nick translation, all probes tagged by the same haptene can be simultaneously labeled in the same vial, thus reducing the procedure to only five separate reactions. The main drawback is that nick translation requires periodical probe DNA isolation. DOP-PCR has the advantage of providing a virtually unlimited source of DNA template, which can be reamplified when needed. The main disadvantage is that only a limited number of probes can be reproducibly labeled by DOP-PCR in the same vial; therefore, labeling requires several separate reactions with each fluorophore. If template DNA from too many probes $(10-15)$ is added in the same vial, PCR-labeling is poor, with many hybridization signals missing. DOPPCR amplification and labeling of the human set of probes resulted in consistent FISH signals mostly when probes were labeled individually. Otherwise results were relatively poor, especially for the cosmids. Nick translation yielded more consistent hybridization signals and was chosen as the labeling method for TM-FISH. Even after nick translation, some cosmid probes still yielded weaker hybridization signals during TM-FISH. To adjust all signals to comparable intensities (Fig. 1, a to c), two to five times higher amounts of labeled DNA from those probes was necessary during hybridizations, compared with P1 or PAC DNA. In the case of mTM-FISH, because the BACs have an average size of about $150 \mathrm{~kb}$, DOP-PCR on sets of 4 to 5 probes/vial could be successfully used as a labeling procedure (Fig. 2, d and f) and yielded robust a hybridization signal. Recent publication of a new human telomere set including BACs and PACs (Knight et al, 2000) should make it possible to apply DOP-PCR labeling for TM-FISH as well.

Whereas the mouse probes were used in a fivecolor assay, for the human probes we developed three different strategies of analysis (Table 1), each with advantages and disadvantages. Laboratories can choose one strategy over the other based on their experience with $\mathrm{FISH}$ procedures, training of their personnel in chromosome identification, and the quality of equipment.

\section{One Set/Five Fluors Approach (1/5)}

In this true M-FISH-like procedure (Table 1), the human or mouse probe sets were labeled using two fluorophores, fluorescein isothiocyanate (FITC) and carboxyrhodamine6G (R6G), and three nonfluorescent haptenes that required fluorescent antibody detection. The haptenes used were dinitrophenyl (DNP, detected with anti-DNP-Cy3.5), biotin (BIO, detected with avidin-Cy5), and digoxigenin (DIG, detected with antiDIG-diethyl aminomethyl coumarin [DEAC]). The combinatorial labeling scheme used (detailed in Fig. 1) results in a 23-color FISH assay, in which the $p$ and $q$ probes of the same chromosome are detected with the same color combination. Because some of the probes yielded very weak signals when in multiple combinations, the assay was made more robust by detecting not only the haptenes but also FITC and R6G with fluorescence-labeled antibodies, thus resembling a five-haptene system. In other words, primary antibodies against fluorescein and rhodamine were detected with secondary antibodies labeled with fluors of similar wavelength (dichlorotriazinylfluorescein [DTAF] for FITC and Cy3 for R6G). Regular M-FISH and TM-FISH were performed on a control case carrying a very small unbalanced translocation (Fig. 1, a and b). The telomeric assay positively identified the translocation in $92 \%$ of all metaphases examined, whereas regular M-FISH analysis identified the origin of the translocated fragment in less than $60 \%$ of metaphases. The reason for this difference is that the painting probes may not be able to detect translocated chromosomal fragments smaller than 2

a to c, TM-FISH analysis of a case with 46, XX add(1)(1q41), based on the "1/5" detection scheme. Using Photoshop (Adobe Systems, Mountain View, California), grayscale images of the same metaphase in FITC (F), rhodamine-Cy3 (R), DNP-Cy3.5 (N), BIO-Cy5 (B), DIG-DEAC (D), and DAPI channels were combined and pseudocolored into two RGB images: $F R$ (including FITC-green, Rhodamine-red, and DAPI-blue) and NBD (including DNP/Cy3.5-red, BIO/Cy5-blue, and DIG/DEAC-green). The arrows in Panels a and $b$ indicate the extra material on 1 qter with a fluorescent signal carrying the color signature of chromosome 8 telomeres. Numbers in Panel a show the positions of all human chromosomes. The two small arrows in the upper-right corner point to the same translocation as detected by M-FISH (very weak signal) or G-banding (the q arm of human chromosome 1). For a better signal visualization, in Panel c, the image of one chromosome of every human pair was split into its original grayscale images. The vertical lines separate the FR from NBD images. The first chromosome in every group as well as the first chromosome after the vertical white line depicts the merged color image as it appears in Panels a and $b$. The white circle indicates the DAPI image of that chromosome. The red, green, or blue dots above each chromosome indicate the pseudo-color assigned for that channel in the respective RGB image (FR or NBD). The same legend appears in the bottom right corner of Panel c. The color chart in Panel d depicts the expected color of every human subtelomeric probe in the FR and NBD images respectively and also indicates (letters) what haptenes (see "Results and Discussion") were used to label each telomere. For example, chromosome 1 telomeric probes were colored red in the FR image and magenta in NBD, indicating that they were labeled by rhodamine (R), DNP (N), and BI0 (B). The "2/3" detection scheme (not shown) can be displayed the same way as in Panels a and b, the only difference being that the two images, FR and NBD, come from different metaphases. The same chart (d) can be used for the mouse TM-FISH, with some exceptions, as also shown in Table 1. Thus, mouse chromosome 5 was labeled FNB (green in the FR and magenta in the NBD images, similar to human 21); chromosome 6 was labeled R (red in the FR image, similar to human 5), and X was labeled N (red in the NBD image, similar to human 20). No probes for the mouse $Y$ chromosome were used. 


\begin{tabular}{|c|c|c|c|c|c|c|c|c|c|c|c|c|c|c|c|c|c|c|}
\hline \multirow[b]{2}{*}{$\mathrm{Chr}$} & \multirow{2}{*}{$\begin{array}{l}\text { Clone } \\
\text { name }\end{array}$} & \multicolumn{5}{|c|}{ Mouse TM-FISH } & \multirow[b]{2}{*}{ Chr } & \multirow{2}{*}{$\begin{array}{l}\text { Clone } \\
\text { name }\end{array}$} & \multicolumn{5}{|c|}{$\begin{array}{c}\text { Human TM-FISH } \\
\text { (1/5 and } 2 / 3 \\
\text { combinations) }\end{array}$} & \multirow[b]{2}{*}{ Chr } & \multicolumn{3}{|c|}{$\begin{array}{c}\text { Human TM-FISH } \\
\text { (3/3 combinations) }\end{array}$} & \multirow[b]{2}{*}{$\operatorname{Set}^{b}$} \\
\hline & & $\mathrm{F}$ & $\mathrm{R}$ & $\mathrm{N}$ & B & $\mathrm{D}$ & & & $\mathrm{F}$ & $\mathrm{R}$ & $\mathrm{N}$ & B & $\mathrm{D}$ & & $D$ & N & B & \\
\hline $1 c$ & $45 \mathrm{C} 1$ & & 1 & 1 & 1 & & $1 p$ & $13 p 11$ & & 1 & 1 & 1 & & $1 p$ & 1 & 1 & 1 & \\
\hline $1 \mathrm{t}$ & 39L15 & & & & & & 19 & $160 \mathrm{~h} 23$ & & & & & & 19 & & & & \\
\hline $2 c$ & $49 N 22$ & 2 & 2 & 2 & & & $2 p$ & $68 j 13$ & 2 & 2 & 2 & & & $4 p$ & & & 4 & \\
\hline $2 \mathrm{t}$ & 20L10 & & & & & & $2 q$ & $210 \mathrm{e} 4$ & & & & & & $4 q$ & & & & \\
\hline $3 c$ & 33D15 & & & 3 & 3 & 3 & $3 p$ & B47a2 & & & 3 & 3 & 3 & $9 p$ & 9 & & 9 & \\
\hline $3 t$ & $443 \mathrm{~K} 17$ & & & & & & $3 q$ & $196 f 4$ & & & & & & $9 q$ & & & & \\
\hline $4 c$ & $39 \mathrm{~B} 18$ & 4 & & & & 4 & $4 p$ & $36 p 21$ & 4 & & & & 4 & $10 p$ & 10 & & & 1 \\
\hline $4 t$ & $362 \mathrm{D} 3$ & & & & & & $4 q$ & cT55 & & & & & & $10 q$ & & & & \\
\hline $5 c$ & $48 \mathrm{H} 24$ & 5 & & 5 & 5 & & $5 p$ & $114 j 18$ & & 5 & & & & $13 q$ & & 13 & 13 & \\
\hline $5 t$ & 40J4 & & & & & & $5 q$ & $240 \mathrm{~g} 13$ & & & & & & $17 p$ & & 17 & & \\
\hline $6 c$ & $45 \mathrm{~K} 20$ & & 6 & & & & $6 p$ & $36 \mathrm{i} 2$ & & 6 & & 6 & 6 & $17 q$ & & & & \\
\hline $6 \mathrm{t}$ & $51 F 24$ & & & & & & $6 q$ & $57 \mathrm{~h} 24$ & & & & & & $20 p$ & & & 20 & \\
\hline $7 c$ & $43 \mathrm{~A} 19$ & & & & 7 & 7 & $7 p$ & $164 \mathrm{~d} 18$ & & & & 7 & 7 & $20 q$ & & & & \\
\hline $7 \mathrm{t}$ & 100021 & & & & & & $7 q$ & $3 \mathrm{k} 23$ & & & & & & $22 q$ & 22 & 22 & & \\
\hline $8 c$ & $20 \mathrm{H} 8$ & 8 & & 8 & & & $8 p$ & $63 \mathrm{~m} 14$ & 8 & & 8 & & & $2 p$ & & 2 & 2 & \\
\hline $8 \mathrm{t}$ & $38 \mathrm{~K} 2$ & & & & & & $8 q$ & $2053 b 3$ & & & & & & $2 q$ & & & & \\
\hline $9 c$ & 23D13 & & & 9 & & 9 & $9 p$ & $34 \mathrm{~h} 2$ & & & 9 & & 9 & $5 p$ & 5 & & & \\
\hline $9 \mathrm{t}$ & $55 \mathrm{~J} 6$ & & & & & & $9 q$ & $112 \mathrm{n} 13$ & & & & & & $5 q$ & & & & \\
\hline $10 \mathrm{c}$ & $47 \mathrm{~K} 7$ & & 10 & & & 10 & $10 p$ & $305 \nmid 4$ & & 10 & & & 10 & $7 p$ & & & 7 & \\
\hline $10 \mathrm{t}$ & $26 \mathrm{C} 12$ & & & & & & $10 q$ & $2136 a 1$ & & & & & & $7 q$ & & & & \\
\hline $11 \mathrm{c}$ & 39E20 & 11 & 11 & & & & $11 p$ & $2209 a 2$ & 11 & 11 & & & & $8 p$ & & 8 & & \\
\hline $11 \mathrm{t}$ & 434L24 & & & & & & $11 q$ & $2072 \mathrm{cl}$ & & & & & & $8 q$ & & & & 2 \\
\hline $12 \mathrm{c}$ & $47 \mathrm{~N} 14$ & 12 & & & 12 & & $12 p$ & $90 i 5$ & 12 & & & 12 & & $11 p$ & 11 & 11 & & \\
\hline $12 \mathrm{t}$ & 34119 & & & & & & $12 q$ & 221k18 & & & & & & $11 q$ & & & & \\
\hline $13 c$ & $50 \mathrm{~K} 9$ & & & 13 & 13 & & $13 q$ & 85a10 & & & 13 & 13 & & $15 q$ & 15 & 15 & 15 & \\
\hline $13 t$ & 3813 & & & & & & $14 q$ & $2006 a 1$ & 14 & & & & & $16 p$ & 16 & & 16 & \\
\hline $14 \mathrm{c}$ & 429N17 & 14 & & & & & $15 q$ & $154 \mathrm{p} 1$ & & 15 & & 15 & & $16 q$ & & & & \\
\hline $14 t$ & 4802 & & & & & & $16 p$ & 119116 & 16 & & 16 & & 16 & $19 p$ & & & 19 & \\
\hline $15 \mathrm{c}$ & $43 \mathrm{G} 16$ & & 15 & & 15 & & $16 q$ & $240 \mathrm{~g} 10$ & & & & & & $19 q$ & & & & \\
\hline $15 t$ & 6212 & & & & & & $17 p$ & $2111 b 1$ & & 17 & 17 & & & $3 p$ & & & 3 & \\
\hline $16 c$ & 5011 & 16 & & 16 & & 16 & $17 q$ & $362 \mathrm{k} 4$ & & & & & & $3 q$ & & & & \\
\hline $16 \mathrm{t}$ & 43D12 & & & & & & $18 p$ & $52 \mathrm{~m} 11$ & & & & 18 & & $6 p$ & 6 & & 6 & \\
\hline $17 \mathrm{c}$ & $65 \mathrm{C} 22$ & & 17 & 17 & & & $18 q$ & $2050 a 6$ & & & & & & $6 q$ & & & & \\
\hline $17 t$ & $50 \mathrm{~F} 18$ & & & & & & $19 p$ & $F 20643$ & & & & & 19 & $12 p$ & 12 & 12 & & \\
\hline $18 \mathrm{c}$ & $53 \mathrm{M} 14$ & & & & 18 & & $19 q$ & F21283 & & & & & & $12 q$ & & & & 3 \\
\hline $18 t$ & $51 \mathrm{~B} 23$ & & & & & & $20 p$ & $2005 a 4$ & & & 20 & & & $14 q$ & & 14 & 14 & \\
\hline $19 \mathrm{c}$ & $26 \mathrm{~B} 5$ & & & & & 19 & $20 q$ & $204 a 16$ & & & & & & $18 p$ & 18 & & & \\
\hline $19 t$ & 49P14 & & & & & & $21 q$ & $63 \mathrm{~h} 24$ & 21 & & 21 & 21 & & $18 q$ & & & & \\
\hline$X_{c}$ & $51 \mathrm{~A} 6$ & & & $x$ & & & $22 q$ & $99 k 24$ & & 22 & 22 & & 22 & $21 q$ & 21 & 21 & 21 & \\
\hline$X t$ & $23 \mathrm{H} 12$ & & & & & & $X Y p$ & $98 c 4$ & xy & & & xy & xy & $X Y p$ & & $x y$ & & \\
\hline & & & & & & & $X Y q$ & $c 8.1 / 2$ & & & & & & $X Y q$ & & & & \\
\hline
\end{tabular}

$\mathrm{Chr}$, chromosome number; $\mathrm{c}$ and $\mathrm{t}$ for mouse clones, the centromeric and telomeric end of the chromosome; $\mathrm{p}$ and $\mathrm{q}$ for human clones, the $\mathrm{p}$ and $\mathrm{q}$ arms of the chromosome; F, FITC; R, R6G; N, DNP; B, BIO; D, DIG.

${ }^{a}$ Numbers within the table indicate the fluors or haptenes with which the probes for the respective chromosomes were labeled. We used numbers so that chromosome identification would be easier. Italic characters of some human clone names indicate cosmid probes. All the other probes were P1, PACs. When human probes are used according to the "2/3" approach, FITC is replaced by BIO and R6G by DIG. The probes in these two columns are then hybridized on one area of the slide, whereas the rest of the human clones (the ones labeled by BIO, DIG, DNP) are hybridized on another area of the slide. Note: To differentiate the chromosomes, information from both hybridizations needs to be combined.

${ }^{b}$ Steps 1 to 3: the three probes mixes used for the "3/3" TM-FISH combination. Probes corresponding to chromosomes 4 and 20 (Mix 1) and chromosomes 7 and 19 (Mix 2), respectively, are differentiated by the size of the fluorescent signal, not by combinatorial labeling.

to $3 \mathrm{Mb}$ (Azofeifa et al, 2000). Thus, FISH with subtelomeric probes appears to be the method of choice for in situ cryptic translocation detection. M-FISH and TM-FISH performed on a set of 14 cytogenetically normal samples from patients with autism did not identify any translocation. More patients are currently under analysis.

The mouse-specific telomeric assay was tested on a cytogenetic sample carrying a translocation. Cells in methanol:acetic acid from three mouse samples (two 
normal and one with translocation) were sent to our laboratory for a blinded study, ie, without disclosing the name of the chromosomes involved. mTM-FISH required only one hybridization on each slide to identify the normal and abnormal samples and the chromosomes involved in the translocation (Fig. 2, $d$ and f), thus confirming the results obtained previously by $\mathrm{G}$ banding.

\section{Two Sets/Three Fluors Approach (2/3)}

This technique uses the same probe combinations as in " $1 / 5$ " (Fig. 1 and Table 1, columns F, R, N, B, and D), but the probes are divided into two groups which are hybridized onto two different $22 \times 22 \mathrm{~mm}$ areas of the same slide. The first group includes a probe set labeled with BIO and DIG (Table 1, columns F, R), whereas the second group consists of a different probe set, labeled with the three haptenes, BIO, DIG, and DNP (Table 1, columns N, B, and D). The reason for replacing the two fluors with haptenes in the first group is to decrease the total number of antibodies used for detection. This is only possible because the two groups of probes are hybridized separately. Thus, antibody detection on both areas of the slide is performed simultaneously using the same mixture of three fluorescence-labeled antibodies, against $\mathrm{BIO}$, DIG, and DNP. It is important to notice that, because of the labeling algorithm (Table 1), neither the first, nor the second group of probe sets can identify the chromosomes by itself, but only in combination. For example, the probes for chromosomes 2 and 11 are both labeled with BIO and DIG in the first group. After hybridization, the color of the subtelomeric regions of chromosomes 2 and 11 will be identical, so this hybridization itself will not detect a $\mathrm{t}(2 ; 11)$. However, in the hybridization on the second area of the slide (using the second group of probes), only the probes for chromosome 2 are again labeled (with DNP). These probes will have the same color as chromosome 20 probes (also labeled with DNP in the second group). However, chromosome 20 probes are not labeled in the first group, and thus chromosomes 2, 11, and 20 can be separated. In conclusion, the combined information from both hybridizations identifies all telomeres. The hybridization signals of the three channels captured from one area and two channels captured from the other area are merged into two separate color images using Adobe Photoshop, and the telomeres are identified by comparisons with the algorithm in the table. Because it uses only three labeled antibodies (and thus only three colors) to detect the haptenes, this procedure is simpler and more robust than " $1 / 5$." The other advantage is that it can be performed on any fluorescence microscope equipped with only three filters.

\section{Three Sets/Three Fluors Approach (3/3)}

This approach was designed for the purpose of providing an assay that does not require sophisticated equipment or personnel trained in chromosome identification. The probes are divided into three sets (Sets
1, 2, and 3, Table 1 and Fig. 2, a to c), each detecting seven or eight chromosomes independently from the other two sets. Probes are labeled with the same three haptenes (BIO, DIG, DNP), and the sets are hybridized on separate areas of the same slide. Combinatorial labeling with three colors yields a maximum of seven combinations, thus allowing simple visual identification of every chromosome, even when a person has no prior training in differentiating human chromosome based on their 4',6-diamidino-2-phenylindole (4') (DAPI) staining. However, based solely on combinatorial labeling, theoretically, only 21 chromosomes can be detected in three independent sets. Because the human complement includes 23 pairs of chromosomes, the remaining two chromosome pairs were detected by ratio labeling (or signal strength), one of them in each of Sets 1 and 2. This was possible, because the telomeric probes of chromosomes 20 (Set 1) and 19 (Set 2) yielded much weaker signals than probes for chromosomes 4 (Set 1) and 7 (Set 2).

\section{Imaging Limitations and Color Display}

For the five-fluor procedure, 1/5, a microscope equipped with five different fluorescence filters and a CCD camera is necessary, whereas for procedures $2 / 3$ and $3 / 3$, any fluorescence microscope equipped with the common three filters is sufficient. In this case, a red, a green, and a blue fluor (aminomethyl coumarin, $[A M C A])$ can be chosen to detect the haptenes, whereas DAPI counterstaining is performed after imaging the FISH signals. Availability of a microscope with four filters allows a choice of three fluors in the visible spectrum and DAPI staining. This scenario makes possible the use of a simple digital camera for image capturing (Henegariu et al, 1999). Although specialized software for chromosome classification based on telomeric signals would be useful, Figures 1 and 2 show that generic imaging software (Photoshop) is sufficient. However, merging and pseudocoloring the grayscale images of all five fluors plus DAPI (as in the 1/5 approach) in Photoshop yielded FISH signals with colors that made it difficult or impossible to differentiate among some of the chromosomes. Simply merging five or six channels in a multicolor Photoshop image does not seem to be a robust enough approach for discriminating the colors of telomere signals, although it was sufficient for chromosome painting probes (Henegariu et al, 1999). The main reason for this is that the signals from the telomere probes are dot-like signals, unequal in size and intensity, that vary significantly even from one metaphase to the other. Therefore, a true 24-color image would require specialized software allowing localized signal enhancement and proportional pseudocoloring. To allow analysis while still using Photoshop, we grouped and pseudocolored the grayscale images of the $1 / 5$ analysis into two triplets: FITC, R6G, and DAPI in a first color image and DEAC, Cy3.5, and Cy5 in a second (Figs. 1 and 2). Merging only three channels (RGB image) allows the hybridization signals to be discriminated by color after simple visual inspection on the 


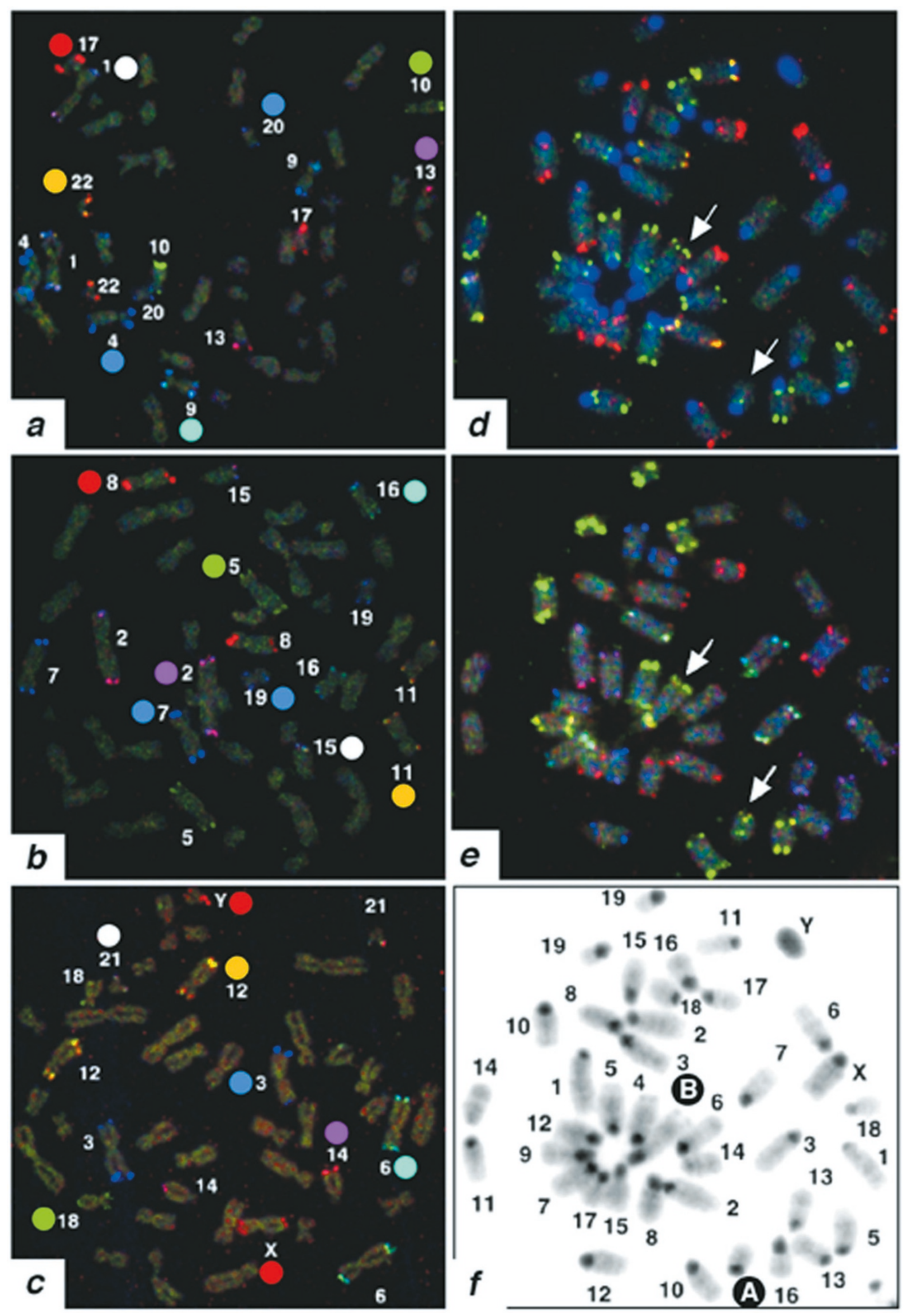

Figure 2. 
computer screen. By comparing the colors of the telomeres in the two images with the provided color chart, all chromosomes (and potential translocations) can be identified.

\section{Materials and Methods}

\section{DNA Probes and Biological Samples}

Probes used to detect the human subtelomeric regions are listed in Table 1 and were previously characterized (National Institutes of Health and Institute of Molecular Medicine, 1996). These probes are located from less than $100 \mathrm{~kb}$ to less than $1000 \mathrm{~kb}$ from the telomeres of their respective chromosomes. All probes are available from ATCC and Research Genetics. The mouse BACs listed in Table 1 were selected using genetic markers that define the centromeric and distal telomeric ends of the Whitehead/MIT recombinational maps of mouse chromosomes (Korenberg et al, 1999). As opposed to the human clones, the precise physical distance of the mouse BAC to the ends of the chromosomes is not yet known. The labeling combinations used for both mouse and human probes are detailed in Table 1.

The various human TM-FISH procedures were performed on several normal control samples, on 14 samples from patients with autism (normal karyotypes), and on a known translocation sample. The latter was from a patient carrying a small translocation on the tip of $1 q$ (46, XX add[1][1q41]), identified cytogenetically. G-banding could not detect the origin of the small translocated fragment. M-FISH and TMFISH were separately used to identify the origin of the translocated fragment. Mouse TM-FISH was performed on two normal cytogenetic samples and on splenocytes from a $12 \mathrm{Gso}$ mouse, known to carry the $(4 ; 9)(B 3 ; D)$ translocation. At the time of the analysis, we did not know which sample contained the translocation or which chromosomes were involved. mTMFISH correctly identified the translocation.

\section{Slide and Probe Preparation and Labeling Strategies}

Slides were prepared according to common cytogenetic procedures, with several modifications aimed at yielding very "flat" nuclei and chromosomes detailed elsewhere (Henegariu et al, 2001b). Briefly, the slide was kept for a few seconds in the hot water vapors of a waterbath at $75^{\circ} \mathrm{C}$. Then a few drops of cell suspension in fixative (3:1 methanol:acetic acid) were pipetted onto the slide. As soon as the fixative started to dry and the cells on the glass surface became visible ("grainy" aspect), four to five drops of acetic acid were quickly placed on the slide and allowed to spread, and then the slide was exposed again for 3 to 4 seconds to the hot water vapors. Then, the slide was quickly dried on a hot metal surface $\left(65-70^{\circ} \mathrm{C}\right)$. The flatness of the cytogenetic preparation was important, because it allowed imaging of all hybridized probes in the same focal plane. Probes were labeled by nick translation or DOP-PCR (Telenius et al, 1992) using dUTP labeled with FITC, R6G, BIO, DIG, and DNP. The labeled dUTPs were custom synthesized in our laboratory by chemical conjugation reactions between reactive allylamine dUTP and succinimidyl ester of fluor/hapetene derivatives (Henegariu et al, 2000). Labeled probe DNA (20 to $100 \mathrm{ng}$ ) were used for FISH, regardless of the method of labeling. Probes yielding weak signals, such as those for human chromosomes $10 p, 19, X$, and $Y$, were used in quantities 2 to 5 times higher than other probes. Probe DNA was prepared by alkaline lysis, using midi/maxiprep kits (Qiagen, Valencia, California; Clontech, Palo Alto, California). The following strategies were used to prepare the probe cocktails for TM-FISH.

Nick Translation Labeling. To initially test the quality of the various human probes, equal amounts of probe DNA (50-100 ng each) for the $p$ and $q$ arms of the first 12 chromosomes were pooled together and labeled into two separate nick translation reactions. The $p$ probes were labeled with $\mathrm{BIO}$ and the $\mathrm{q}$ probes with DIG, then mixed together, precipitated, and hybridized on a normal control slide. After hybridization and antibody detection, the chromosomes were examined for the presence or absence of signals. Particular notice was taken of the probes yielding weak signals (the strength of a signal did not need precise quantification, only visual approximation). The same strategy was used to test the probes for the remaining chromosomes. Once this information was gathered, probe cocktails were prepared. As an example, for human $1 / 5$ strategy, all probes to be labeled with a given haptene- or fluor-dUTP (Table 1) were pooled together for a total of five probe cocktails. In every such pool, the probes yielding good signals were added at about 20 to $50 \mathrm{ng} /$ hybridization, whereas 2 to 5 times more DNA was necessary for the probes found previously to yield weak signals. The total amount of DNA to be labeled was multiplied by the number of slides desired. In nick translation, there was no limit in reaction volume or amount of probe simultaneously labeled. The reaction worked equally well in a 15 or $50 \mathrm{ml}$ tube

\footnotetext{
a to $\mathrm{c}$, The human $3 / 3$ detection scheme, using all telomere probes combined in three sets: 1 (a), 2 (b), and 3 (c). Probes were labeled with biotin (BI0), digoxigenin (DIG), and dinitrophenyl (DNP); thus there were only three initial colors (red, green, and blue, identical to the NBD images in Figure 2). The colored dots indicate the expected color of every chromosome pair in each of the three sets and provide the labeling scheme for every chromosome. Red is DNP-Cy3.5 (N), green is DIG-DEAC (D), blue is BIO-Cy5 (B), cyan is BD, magenta is NB, yellow is BD, and white is NBD. Note that in the $3 / 3$ detection scheme, each probe set detects the chromosomes independently from the other two sets. For chromosomes 20 in Set 1 (a) and 19 in Set 2 (b), we used ratio labeling for detection. The telomeric probes for these two chromosomes yield signals significantly smaller than the signals for chromosomes 4 (a) and 7 (b), respectively. This obvious signal difference, allows chromosome identification. $d$ to $f$, Mouse TM-FISH images ( $1 / 5$ strategy). Because all mouse chromosomes are acrocentric, only one probe is close to the telomere; the other one is close to the centromere. Images were prepared as described for the human probes in Figure 2, with FR (in d) and NBD (in e). Arrows point toward the two chromosomes carrying the reciprocal $t(4 ; 9)$ translocation. The reverse DAPI image of the same metaphase is also shown (e). The white upper-case letters indicate the positions of the derivative chromosomes $4(A)$ and $9(B)$ in the metaphase.
} 
incubated at $15^{\circ} \mathrm{C}$ in a waterbath. For any one hybridization, aliquots of DNA from each of the five labeled pools were mixed together, ethanol precipitated in the presence of 40 to $50 \mu \mathrm{g}$ Cot-1 DNA (Life Technologies, Rockville, Maryland), resuspended in hybridization buffer, and hybridized overnight under a $22 \times 22$ $\mathrm{mm}$ coverslip. After antibody detection and imaging, all signals were carefully accounted for. If, for example, the signal of a FITC-labeled probe was weak or missing, a separate nick translation reaction using FITC-dUTP was performed for that probe and then tested and mixed with the initial FITC-labeled pool. This strategy allowed convenient "repairing" of every probe cocktail made by nick translation.

DOP-PCR Labeling. This technique was applied to the mouse clones. Using a known degenerate primer (Telenius et al, 1992), initial PCR reactions at low stringency were separately performed on every probe DNA. The PCR cycling included annealing temperatures of $15^{\circ} \mathrm{C}$ in the first cycle, $30^{\circ} \mathrm{C}$ in the next four cycles, and $54^{\circ} \mathrm{C}$ for the remaining 25 cycles (Henegariu et al, 1999). For every BAC used, this DOP-PCR amplification provided the "PCR template" for the subsequent PCR labeling reactions. In our hands, mixing in the same tube the PCR templates of more than four to five probes for PCR labeling resulted in a decrease of the hybridization signals. To prevent this, separate PCR reactions with any labeled dUTP were performed on one or, at the most, two probes per vial. Although this approach required dozens of labeling reactions, it preserved probe complexity and allowed for reproducibility of hybridization signals. PCR amplification eliminated the need for subsequent probe preparations by alkaline lysis. As a guideline, we used 3 to $4 \mu \mathrm{l}$ "PCR template" for any $100 \mu \mathrm{l}$ PCR labeling reaction. If two probes were labeled in the same vial, we used $3 \mu$ l of each "PCR template." Labeled PCR product (4-5 $\mu$ l per probe) was used for one hybridization. (For example, for mouse chromosome 1 [Table 1], probes 1c and 1t were labeled in the same vials; 8 $\mu$ l each of R6G-, DNP-, and BIO-labeled PCR products of $1 c+1 t$ were used for one hybridization.)

\section{Antibody Detection, Imaging, and Image Analysis}

All antibodies used for detection were stored as 1 $\mathrm{mg} / \mathrm{ml}$ stock solutions and were used at 1:100 dilutions, in $100 \mu \mathrm{l} 4 \times \mathrm{SSC} / \mathrm{slide}$. All antibody incubations were done for 10 minutes at $37^{\circ} \mathrm{C}$ and were followed by 10 - to 15 -minute washes in $4 \times$ SSC/0.1\% Tween20 at 37 to $42^{\circ} \mathrm{C}$. Some antibodies were purchased labeled; others were custom labeled in our laboratory, using standard protocols (Molecular Probes, Eugene, Oregon; Amersham-Pharmacia Biotech, Piscataway, New Jersey). When only BIO, DIG, and DNP were used to label probes, the detection required only one layer of antibody (one-step detection), including mouse anti-DIG-FITC (Sigma, St. Louis, Missouri), avidin-Cy5, and rat anti-DNP (Accurate Chemical, Westbury, New York) custom labeled with Cy3.5 (Pharmacia-Amersham). For the more complex " $1 / 5$ " scheme, several tests were done with antibodies from several manufacturers to find a combination of proteins that did not cross-react nonspecifically with one another. These experiments resulted in the following detection/amplification scheme: FITC was detected with goat anti-FITC followed by donkey antigoat-DTAF (Accurate Chemical). Rhodamine was detected with rabbit antirhodamine (Molecular Probes) custom-conjugated with Cy3 (Pharmacia-Amersham) followed by donkey antirabbit (Accurate Chemical) custom-conjugated with $\mathrm{Cy} 3$. BIO was detected with one layer of Avidin-Cy5. DNP was detected with rat antiDNP-Cy3.5. DIG was detected with sheep antiDIG (Boehringer Mannheim, Indianapolis, Indiana) custom conjugated with DEAC (Molecular Probes) followed by donkey antisheep (Accurate Chemical) custom conjugated with DEAC. All antibodies were combined into three detection steps/vials. In the first step, we mixed in the same vial goat anti-FITC, rabbit antirhodamine-Cy3, sheep anti-DIG-DEAC, and rat anti-DNP-Cy3.5. As our available goat anti-FITC was binding nonspecifically biotin, we added $1 \mu \mathrm{l}$ of a $1 \mathrm{~mm}$ custom-made BIO-dUTP to the antibody mix immediately before using the antibodies. After 10 minutes antibody incubation and 10 to 15 minutes postantibody wash, the slide was subjected to the second step of detection. This included donkey antigoatDTAF and donkey antirabbit-Cy3. After the usual 10 minutes incubation and 10 to 15 minutes wash, a third layer of detection was added (third step), which included avidin-Cy5 and donkey antisheep DEAC. To block the weak nonspecific binding of our donkey antisheep to the goat anti-FITC antibody used at the first layer, $1 \mu \mathrm{l}$ of a $1 \mathrm{mg} / \mathrm{ml}$ goat IgG solution was added in the same vial with avidin and donkey antisheep before using them. After incubation and washing, the slide was stained with DAPI, mounted with antifade solution, and examined at the microscope using appropriate filters (Henegariu et al, 2000). Gray-scale images of every channel were captured on an Olympus Provis microscope (Olympus, Tokyo, Japan), equipped with a Photometrix Sensys camera, using the PowerGene M-FISH software (Perceptive Scientific Instruments, Inc., League City, Texas). Grayscale images were pseudocolored in groups of three in Adobe Photoshop (creating RGB images). In the first combination (FR), FITC was pseudocolored green, rhodamine/Cy3 red, and DAPI blue. In the other combination (NBD), DNP-Cy3.5 was pseudocolored red, BIO-Cy5 blue, and DIG-DEAC green.

\section{References}

Azofeifa J, Fauth C, Kraus J, Maierhofer C, Langer S, Bolzer A, Reichman J, Schuffenhauer S, and Speicher MR (2000). An optimized probe set for the detection of small interchromosomal aberrations by use of 24 -color FISH. Am J Hum Genet 66:1684-1688.

Bacino CA, Kashork CD, Davino NA, and Shaffer LG (2000). Detection of a cryptic translocation in a family with mental retardation using FISH and telomere region-specific probes. Am J Med Genet 92:250-255. 
Ballif BC, Kashork CD, and Shaffer LG (2000). FISHing for mechanisms of cytogenetically defined terminal deletions using chromosome-specific subtelomeric probes [In Process Citation]. Eur J Hum Genet 8:764-770.

Flint J, Wilkie AO, Buckle VJ, Winter RM, Holland AJ, and McDermid HE (1995). The detection of subtelomeric chromosomal rearrangements in idiopathic mental retardation. Nat Genet 9:132-140.

Ghaffari SR, Boyd E, Tolmie JL, Crow YJ, Trainer AH, and Connor JM (1998). A new strategy for cryptic telomeric translocation screening in patients with idiopathic mental retardation. J Med Genet 35:225-233.

Granzow M, Popp S, Keller M, Holtgreve-Grez H, Brough M, Schoell B, Rauterberg-Ruland I, Hager HD, Tariverdian G, and Jauch A (2000). Multiplex FISH telomere integrity assay identifies an unbalanced cryptic translocation $\operatorname{der}(5) \mathrm{t}(3$; 5)(q27;p15.3) in a family with three mentally retarded individuals. Hum Genet 107:51-57.

Henegariu O, Bray-Ward P, and Ward DC (2000). Custom fluorescent-nucleotide synthesis as an alternative method for nucleic acid labeling. Nat Biotechnol 18:345-348.

Henegariu O, Dunai J, Chen X-N, Korenberg JR, Ward DC, and Greally JM (In press, 2001a). A triple color FISH technique for mouse chromosome identification. Mamm Genome.

Henegariu O, Heerema NA, Bray-Ward P, and Ward DC (1999). Colour-changing karyotyping: An alternative to MFISH/SKY [letter]. Nat Genet 23:263-264.

Henegariu O, Heerema NA, Wright LL, Bray-Ward P, Ward DC, and Vance GH (In press, 2001b). Improvements in cytogenetic slide preparation: Controlled chromosome spreading, chemical aging and gradual denaturing. Cytometry.

Knight SJ, Horsley SW, Regan R, Lawrie NM, Maher EJ, Cardy DL, Flint J, and Kearney L (1997). Development and clinical application of an innovative fluorescence in situ hybridization technique which detects submicroscopic rearrangements involving telomeres. Eur J Hum Genet 5:1-8.
Knight SJ, Lese CM, Precht KS, Kuc J, Ning Y, Lucas S, Regan R, Brenan M, Nicod A, Lawrie NM, Cardy DL, Nguyen $\mathrm{H}$, Hudson $\mathrm{TJ}$, Riethman $\mathrm{HC}$, Ledbetter $\mathrm{DH}$, and Flint $\mathrm{J}$ (2000). An optimized set of human telomere clones for studying telomere integrity and architecture. Am J Hum Genet 67:320-332.

Knight SJ, Regan R, Nicod A, Horsley SW, Kearney L, Homfray T, Winter RM, Bolton P, and Flint J (1999). Subtle chromosomal rearrangements in children with unexplained mental retardation (see comments). Lancet 354:1676-1681.

Korenberg JR, Chen XN, Devon KL, Noya D, Oster-Granite ML, and Birren BW (1999). Mouse molecular cytogenetic resource: 157 BACs link the chromosomal and genetic maps. Genome Res 9:514-523.

National Institutes of Health and Institute of Molecular Medicine (1996). A complete set of human telomeric probes and their clinical application. (Published erratum appears in Nat Genet, 1996, 14:487.) Nat Genet 14:86-89.

Schrock E, du Manoir S, Veldman T, Schoell B, Wienberg J, Ferguson-Smith MA, Ning Y, Ledbetter DH, Bar-Am I, Soenksen D, Garini Y, and Ried T (1996). Multicolor spectral karyotyping of human chromosomes (see comments). Science 273:494-497.

Slavotinek A, Rosenberg M, Knight S, Gaunt L, Fergusson W, Killoran C, Clayton-Smith J, Kingston H, Campbell RH, Flint J, Donnai D, and Biesecker L (1999). Screening for submicroscopic chromosome rearrangements in children with idiopathic mental retardation using microsatellite markers for the chromosome telomeres. J Med Genet 36:405-411.

Speicher MR, Gwyn Ballard S, and Ward DC (1996). Karyotyping human chromosomes by combinatorial multi-fluor FISH. Nat Genet 12:368-375.

Telenius H, Carter NP, Bebb CE, Nordenskjold M, Ponder BA, and Tunnacliffe A (1992). Degenerate oligonucleotideprimed PCR: General amplification of target DNA by a single degenerate primer. Genomics 13:718-725. 